\title{
Evaluación de la enseñanza-aprendizaje en el aula y percepción del estudiante de educación superior
}

\section{Assessment of Teaching-Learning in the Classroom and Student Perception of Higher Education}

Cirilo Segovia Suller*

http://dx.doi.org/10.21503/lex.v16i22.1661

* Magíster en Educación. Docente de la Facultad de Derecho y Ciencia Política de la Universidad Alas Peruanas.

Correo electrónico: ciriloseg@yahoo.com.mx

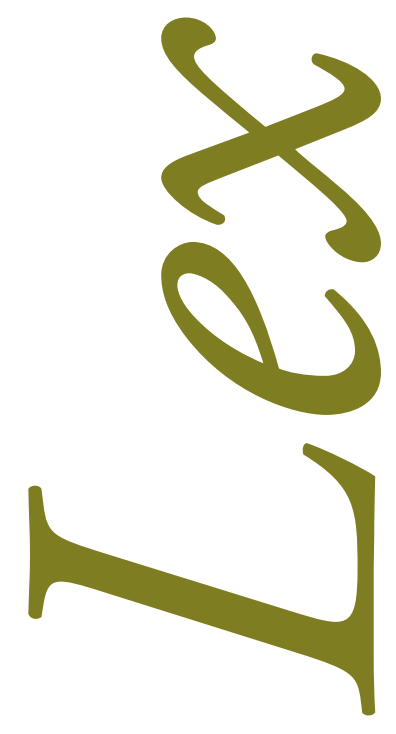
(c) (C) Los autores. Artículo publicado por la Revista Lex de la Facultad de Derecho y Ciencias Políticas de la Universidad Alas 


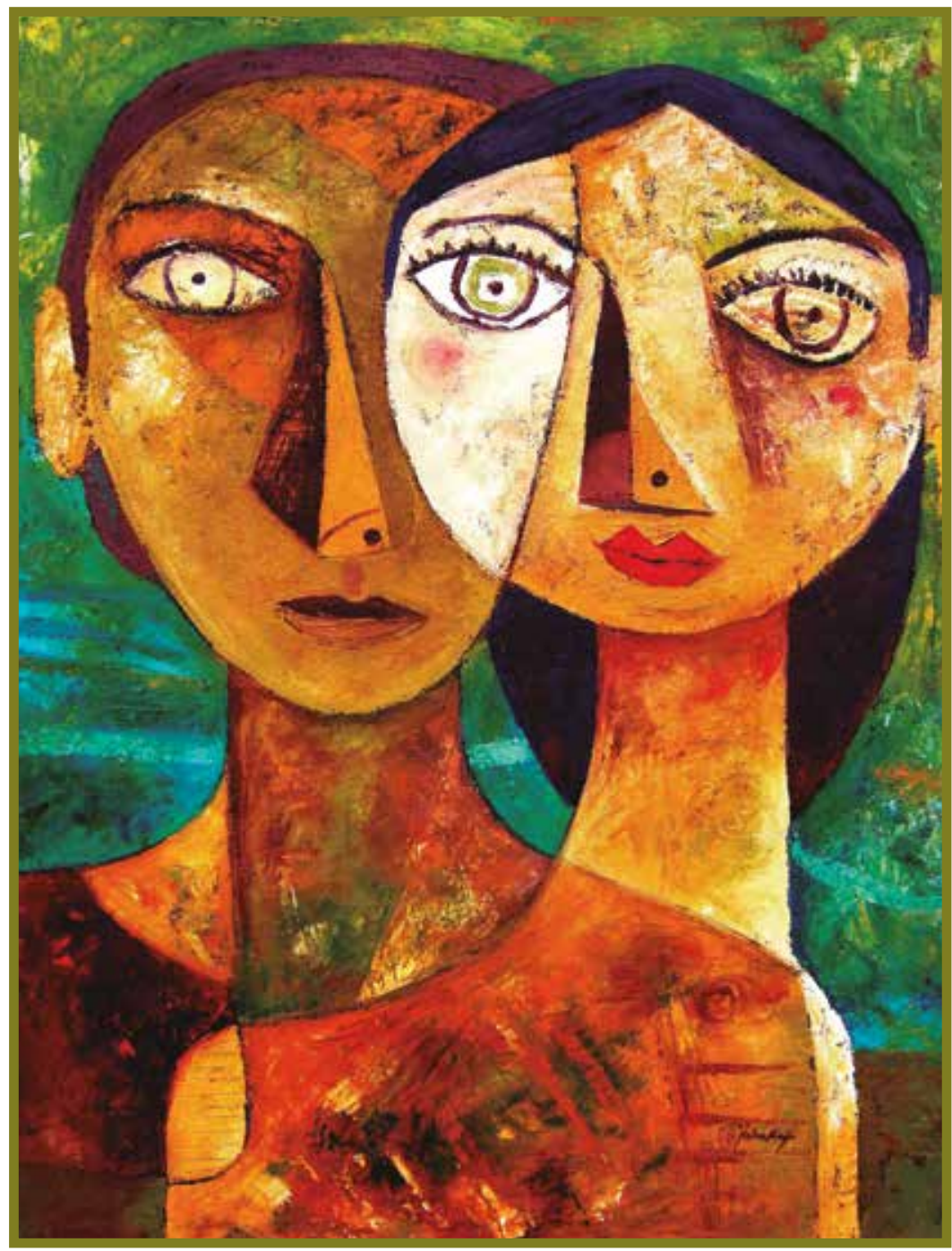

Miradas. Pintura. Juan Carlos Náanake. 


\section{RESUMEN}

En una asignatura que genera cierto grado de dificultad en la enseńanza del docente y el aprendizaje del estudiante, es recomendable que el docente utilice estrategias didácticas que favorezcan el proceso de "enseñar a aprender" y el estudiante utilice estrategias de "aprender a aprender". Por tal motivo, se investigó en qué medida la evaluación de la enseñanza-aprendizaje en el aula es explicada por la percepción del estudiante de educación superior, el mismo que definió el objetivo de evidenciar si la evaluación de la enseńanza-aprendizaje en el aula es explicada por la percepción del estudiante de educación superior. Para luego formular la hipótesis de que la evaluación de la enseñanza-aprendizaje en el aula es explicada por la percepción del estudiante de educación superior. La investigación fue aplicada, explicativa y no experimental. El enfoque fue cuantitativo y el método hipotético-deductivo. De igual manera fue observacional, prospectiva, transversal y analítica. Se validó el instrumento para identificar, definir y medir el constructo. Se aplicó el alfa Cronbach para determinar la consistencia interna del instrumento, la correlación de Rho de Spearman para medir la asociación entre las variables, se calculó el p- valor para contrastar la hipótesis, y se procedió al análisis factorial confirmatorio para explicar la relación causal entre las variables. La conclusión fue que la evaluación de la enseñanza-aprendizaje en el aula es explicada significativamente por la percepción del estudiante de educación superior.

Palabras clave: evaluación de la enseñanza-aprendizaje en el aula, percepción del estudiante, planificación, ejecución y evaluación de la sesión de clases, momentos de la sesión de clases.

\section{ABSTRACT}

For a subject generating a certain degree of difficulty in the teaching from the teacher and the learning of the student, it is recommended that the teacher uses didactic strategies to favor the "teaching to learn" process and that the student uses "learning to learn" strategies. For this reason aninvestigation about to what extent is the evaluation of the classroom teaching-learning process explained by the perception of the higher education student, was carried out. The same, which defined the objective of showing whether the evaluation of the teaching-learning in the classroom is explained by the perception of the higher education student, to, afterwards, formulate the hypothesis that the evaluation of the teaching-learning in the classroom is explained by the perception of the higher education student. The research was applied, explanatory and non-experimental. The approach was quantitative and the hypothetical-deductive method was applied. The investigation was also observational, prospective, transversal and analytical. The instrument to identify, define and measure the construct was validated. Alpha Cronbach was applied to determine the internal consistency of the instrument; Spearman's rank correlation coefficient was 
applied to measure the association between variables; $\mathrm{p}$ - value was calculated to contrast the hypothesis; and confirmatory factorial analysis was applied to explain the cause-effect relation between the variables. The conclusion was that the evaluation of teaching-learning in the classroom is significantly explained by the perception of the higher education student.

Key words: assessment of teaching-learning in the classroom, student perception, planning, execution and evaluation of the class session, moments of the class session. 


\section{INTRODUCCIÓN}

En los meses de marzo a junio de 2018, ochenta y siete (87) estudiantes del décimo ciclo de la Facultad de Derecho y Ciencia Política de la Universidad Alas Peruanas se matricularon en la asignatura Métodos Estadísticos para la Investigación; aula 201 con 23 estudiantes; aula 202 con 35 estudiantes; y aula 302 con 29 estudiantes. Estudiantes que procedieron a evaluar el proceso de enseñanza-aprendizaje del docente a cargo, desde su percepción sobre las vivencias y experiencias ocurridas en los momentos de la sesión de clases del ciclo académico concluido.

Si bien existen diversas opiniones entre los docentes respecto a si los estudiantes están o no en la capacidad de evaluar la calidad del servicio educativo que reciben en el aula, resulta necesario considerar que el estudiante es el usuario o cliente principal del servicio educativo, sea que este se ofrezca en una institución pública o privada; al mismo tiempo, sus vivencias y experiencias sobre el proceso de enseñanza-aprendizaje en el aula son de mucha importancia para evaluar el desempeńo de un docente. Solo así se puede entender la importancia de la percepción del estudiante para diagnosticar la gestión en el aula, y de realizar el análisis FODA del docente para mejorar la calidad del proceso de enseñanza-aprendizaje de la institución de educación superior, sea pública o privada.

En ese orden de ideas, se trata de aprovechar la experiencia de ser evaluado en forma anónima por los estudiantes (usuarios o clientes) a fin de mejorar la gestión de las sesiones de clases; se trata de diagnosticar las fortalezas y debilidades didácticas del docente en el aula; de aprovechar la oportunidad de ser mejor docente convirtiendo la debilidad en fortaleza; de aprovechar la amenaza del entorno externo (desarrollo indetenible de la ciencia, la tecnología de la comunicación e información, y la generación creciente de un espacio on line de la educación) y convertirla en una oportunidad para adecuar la labor docente a los retos del siglo XXI; pero sobre todo, para corregir los errores que los estudiantes detectan en la planificación, ejecución y evaluación de la enseńanza-aprendizaje de la asignatura de la que es responsable el docente. 
De tal manera que se puede afirmar que la percepción del estudiante nos brinda la oportunidad de ser mejores en la planificación y organización de las sesiones de clases; de ser mejores en la ejecución de la enseńanza- aprendizaje en el aula; así como de ser mejores en la evaluación cognitiva, procedimental y actitudinal del estudiante de educación superior. La finalidad, sin duda, es mejorar la calidad del servicio educativo que se brinda en el aula, en la medida que es el escenario donde el estudiante se forma como un futuro profesional del país.

En ese orden, el artículo se orienta a estudiar la experiencia vivida por los estudiantes de la asignatura Métodos Estadísticos para la Investigación que se llevó a cabo en el período 2018I. El mismo que generó reflexiones sobre cómo lograr que el dominio del docente sobre el contenido de la asignatura se transfiera en forma fluida, sencilla y motivadora al estudiante, cómo mejorar la planificación de la sesión de clase de acuerdo al saber previo y el contenido silábico, cómo mejorar la ejecución de la estrategia de enseńanza-aprendizaje en el aula, y cómo mejorar la evaluación a fin de reforzar el aprendizaje cognitivo, procedimental y actitudinal del estudiante.

Se trata, en todo momento, de lograr una planificación de la asignatura con una orientación formativa ordenada, secuencial y organizada. Se trata de que el docente mejore la estrategia de enseńanza-aprendizaje a fin de que la asignatura sea comprensible, agradable y motivadora. Se trata de mejorar la ejecución de las actividades en los distintos momentos de la sesión de clase, a fin de que el estudiante logre un aprendizaje significativo del contenido de la asignatura. Se trata de mejorar la evaluación que refuerce el aprendizaje cognitivo, procedimental y actitudinal. Se trata, sobre todo, de mejorar la calidad del servicio educativo que el docente brinda al estudiante de educación superior en el aula.

Finalmente, el propósito de una institución de educación superior es: 1) Mejorar la calidad de la enseñanza que el docente brinda en el aula; 2) Mejorar la calidad del aprendizaje del estudiante, que se convertirá en el futuro profesional del país; y, 3) Mejorar la calidad de la gestión administrativa-tecnológica que brinda la institución educativa a través de los funcionarios y empleados. Es en este contexto que actúan los tres actores responsables de mejorar la calidad del servicio educativo que brindan las universidades.

\section{ALGUNAS SUPOSICIONES NECESARIAS}

¿Por qué es importante recoger la opinión de los estudiantes mediante un cuestionario? Porque es el principal usuario o cliente del servicio educativo que brindan las instituciones educativas públicas y privadas; y porque el impacto sociocultural de su aprendizaje profesional repercutirá en el desarrollo de la ciencia, tecnología y el progreso sustentable y sostenible de un país. Porque el estudiante es el principal protagonista del escenario (el aula), donde se evidencia con objetividad la calidad del servicio educativo que recibe de una institución edu- 
cativa. Porque el estudiante es la razón de ser de una institución de educación superior, donde se forman los futuros profesionales del país y sus logros promoverán el prestigio de la calidad educativa de la institución educativa de origen. En consecuencia, todo lo que se relaciona con la enseñanza y el aprendizaje debe ser entendida como la ciencia y la esencia de un docente de educación superior.

¿Cuál debe ser la finalidad de una encuesta que se desarrolla en las aulas de una institución educativa? La finalidad es evidenciar la calidad educativa que se imparte en el aula, escenario donde se brinda el servicio educativo al estudiante. Para ello es necesario recoger los hechos ocurridos en el aula, estimulando al estudiante a expresar su vivencia y experiencia del ciclo académico; y obtener datos objetivos, confiables y fiables del principal protagonista del escenario educativo: el estudiante.

¿Cuál debe ser la actitud del docente respecto a la evaluación de su labor educativa desde la percepción de estudiante? Es importante que el docente asuma la evaluación de su labor educativa, como una oportunidad para mejorar la ciencia y el arte de enseñar en el aula. Que la evaluación es un proceso formativo que debe impulsar el docente para cambiar las tradicionales concepciones y modelos educativos de enseñanza y aprendizaje; por las modernas concepciones y modelos flexibles, producto del avance indetenible de la ciencia y la tecnología en el ámbito de la educación. En ese entendido, la encuesta es una forma de democratizar la opinión del estudiante, a fin de que pueda expresar su satisfacción o no acerca del servicio educativo que recibe; es un puente de comunicación entre el docente y el estudiante que ayuda a identificar las fortalezas y debilidades del docente, y permite identificar las amenazas y oportunidades que se generan en el entorno interno y externo de la institución educativa.

¿Por qué es importante implementar la encuesta en el aula de clases? Porque el aula es el escenario natural donde se pone en evidencia el proceso didáctico del docente que enseńa a aprender y del estudiante que aprende a aprender. Sobre el particular, Mejía Mejía Elías ${ }^{1}$ señala que "en la investigación pedagógica, contrariamente a lo que muchos piensan, el campo de trabajo es el aula, la institución educativa, y no la comunidad". Es decir, el aula y la institución educativa son los escenarios de trabajo donde el investigador debe recoger las evidencias empíricas, las vivencias y experiencias de los docentes, estudiantes, empleados y funcionarios para realizar una investigación educativa formativa, científica con proyección social de la Universidad.

1 Elías Mejía Mejía, Metodología de la investigación científica, 1a ed. (Lima: Universidad Nacional Mayor de San Marcos, Facultad de Educación, Unidad de Postgrado, 2005), 39. 


\section{MARCO TEÓRICO}

\subsection{El proceso de enseñanza-aprendizaje}

¿Qué es la didáctica? La pedagogía es la ciencia que estudia a las personas que se forman como profesionales de la educación. La didáctica es una parte de la pedagogía que se encarga de todo lo relacionado con la enseñanza y el aprendizaje. Decía Juan Amos Comenius, pedagogo humanista y filósofo checo, que el buen docente no es calificado por sus logros académicos, sino por el logro de sus estudiantes. En esa lógica, no se trata de que el docente demuestre sus conocimientos académicos en el aula, sino de que demuestre cómo enseña al estudiante a aprender los conocimientos académicos que posee de la asignatura a su cargo. Por lo mismo, se dice que la didáctica es la ciencia y esencia del docente para gerenciar y poner en evidencia su competencia en el arte de enseñar a aprender al estudiante.

En este contexto, es necesario referirnos a María Inés Solar R. y Claudio Díaz L., ${ }^{2}$ quienes señalan que

uno de los grandes desafíos de los docentes es encontrarle significado a su experiencia pedagógica. El rol de las teorías, las prescripciones externas y las experiencias de otros docentes adquiere relevancia cuando ellas pueden articularse con la experiencia del docente $y$, a su vez, tengan sentido en su trabajo. No en vano los formadores de docentes y los investigadores buscan comprender la forma cómo las instituciones educativas median y transforman el 'qué y 'cómo' enseñan los docentes, con el fin de conocer sus procesos de aprendizaje.

Cabe preguntar también cuáles son los componentes de la didáctica. Los componentes de la didáctica son la matética, la sistemática y la metódica. ¿Qué es la matética? Es aquella parte de la didáctica que estudia al ser que aprende y ayuda a identificar los tipos de estudiantes. ¿Qué es la sistemática? Se refiere a la estructura de la sesión de clases, en la medida que una clase sin estructura es similar a un barco que navega sin rumbo. ¿Qué es la metódica? Es la manera, forma y estilo de cómo el docente brinda la sesión de clase a los estudiantes. En ese sentido, es necesario referirnos a María Inés Solar R. y Claudio Díaz L., ${ }^{3}$ quienes concluyen que:

... si los académicos están involucrados activamente en la reflexión de lo que sucede en sus aulas, ellos están en posición de descubrir si existe alguna brecha entre lo que enseñan y lo que los estudiantes aprenden. La premisa fundamental de la investigación de los procesos de enseñanza - aprendizaje es, sin duda, que los docentes deberían utilizar sus aulas como laboratorios para estudiar el proceso de aprendizaje. Los docentes deberían convertirse en observadores expertos y sistemáticos acerca de cómo sus estudiantes aprenden.

2 María Inés Solar R. y Claudio Díaz L., "Los procesos de enseńanza-aprendizaje en el aula universitaria: una mirada desde las creencias de académicos de trabajo social y periodismo", Estudios Pedagógicos XXXV, n. o 1 (2009): 182.

3 Ibid., 195-196. 
Esto nos lleva a la necesaria pregunta de qué es el proceso de enseñanza-aprendizaje en el aula. El proceso de enseńanza-aprendizaje en el aula se concibe como el espacio en el cual el principal protagonista es el estudiante, y el docente cumple con la función de facilitar los procesos de aprendizaje. En ese sentido, el aprendizaje es el proceso a través del cual el estudiante adquiere el conocimiento teórico, la habilidad procedimental y la actitud conductual con valores como resultado de su estudio, experiencia, formación, razonamiento y observación.

Por lo tanto, es necesario referirnos a Santos Soubal Caballero, ${ }^{4}$ quién señala lo siguiente:

El aula en las que se realizan diversas actividades pedagógicas hay que verla como una realidad que se vive en cada momento, en que los sujetos construyen los conocimientos, es una continuidad de su vida cotidiana, no ideal que se forma para las horas en que el alumno está en la escuela. Esto último es una de las razones, entre otras, que profundiza la brecha entre la educación y sociedad.

En consecuencia, la investigación llevada a cabo se focaliza en el estudio de una parte de la sistemática, es decir, la actuación del docente en las fases de planificación, ejecución y evaluación en el aula. De igual manera, la percepción del estudiante se focaliza en los momentos de inicio, proceso y salida de la sesión de clases. En ese orden, la investigación se desarrolló entre marzo a junio de 2018, tiempo en que el docente desarrolló la labor de enseñar al estudiante a aprender, y la tarea del estudiante consistió en aprender a aprender el contenido silábico de la asignatura.

\subsection{Percepción del estudiante}

Un concepto que debe precisarse es qué entendemos por percepción del estudiante. Sobre el particular, se señala que la percepción es la manera en que el cerebro del estudiante siente las sensaciones que recibe a través de los sentidos para formarse una impresión consciente de la realidad física de su entorno (el aula e institución educativa). En ese orden, el cerebro del estudiante describe el conjunto de procesos mentales mediante el cual selecciona, organiza e interpreta la información proveniente de estímulos, pensamientos, sentimientos y vivencias a partir de su experiencia educativa en el ciclo académico, de una manera lógica y significativa.

En el contexto de la presente investigación, ¿qué percepción del estudiante interesa estudiar? Interesa poner énfasis en el contexto del aula donde ocurren los tres momentos de una sesión de clases: la actividad de inicio, la actividad de proceso y la actividad de salida. Al respecto, Imbernon Francesc ${ }^{5}$ señala que

4 Santos Soubal Caballero, "La gestión del aprendizaje. Algunas preguntas y respuestas sobre en relación con el desarrollo del pensamiento en los estudiantes", Polis 7, n. ${ }^{\circ} 21$ (2008): 325.

5 Francesc Imbernon, Mejorar la enseñanza y el aprendizaje en la Universidad, $1^{\text {a }}$ ed. (Barcelona, España: Ediciones Octaedro, 2009), 13 
una clase es como una novela o, si se prefiere, como una narración corta o un relato que hacemos constantemente: hay un principio que te introduce en el tema, que te sitúa en el contexto y que te motiva a seguir; un desarrollo que te introduce en la trama importante del relato y una salida o desenlace que te lleva al final de la lectura, a una conclusión.

En tal circunstancia, Imbernon Francesc ${ }^{6}$ también precisa, respecto a la actividad de inicio, que

una buena clase empieza con un buen principio. El que escucha siempre necesita claramente una introducción bien estructurada que le lleve a "sintonizar" con el profesor o profesora y con el tema que éste pretende desarrollar. Y para sintonizar necesitamos pensar algo que llame la atención del grupo y lo motive a escuchar, que el alumnado se haga un buen esquema mental (una evocación) o escrito de lo que estamos trabajando, y si lo que decimos y trabajamos tiene una utilidad; es posible que la sintonía con ellos sea más adecuada.

Al respecto, Eduardo M. Pacheco Peña y Cosme Sanyorei Porras ${ }^{7}$ señalan que la actividad de inicio debe ser entendida como un proceso de mejora continua en la organización planificada de las sesiones de clases en el aula, que tenga en cuenta la problematización planeada desde la realidad del estudiante; la motivación inicial que capte la atención inicial del estudiante; la revisión de los saberes previos del estudiante; el conflicto cognitivo que debe generar un debate entre los estudiantes; y el propósito y organización que implica dar a conocer los aprendizajes que se espera que logren, el tipo de actividad que van a realizar y cómo serán evaluados.

De igual manera, agrega Francesc Imberno ${ }^{8}$ respecto a la actividad de proceso:

Es la parte central de la exposición. En ella desarrollamos el tema académico, las ideas principales y las secundarias. Pero no podemos únicamente dedicarnos a explicar sin tener en cuenta el auditorio. Es fácil comprobar que no todos aprendemos de igual forma, aunque el mensaje sea el mismo. Hemos de realizar un desarrollo allí donde podamos ir comprobando si todos aprenden lo que estamos enseñando, reflexionando en la acción para analizar si se sigue el discurso, si tenemos que matizarlo, poner un ejemplo, un dato, hacer preguntas, parar... el desarrollo puede contener muchos elementos que nos ayudan a que el alumno aprenda aquello que estamos enseńando.

$6 \quad$ Ibid., 13-14.

7 Eduardo M. Pacheco Peńa y Sanyorei Porras Cosme, "Los momentos de la sesión a través de las rutas de aprendizaje. Propuesta de trabajo para los alumnos de las carreras de Educación Secundaria de la UNDAC”, Horizonte de la Ciencia 4, n. 7 (diciembre 2014): 79-81.

8 Francesc Imbernon, op. cit., 15-16. 
Sobre el particular, Eduardo M. Pacheco Peña y Sanyorei Porras Cosme9 señalan que la actividad de proceso debe ser entendida como un proceso de mejora continua de la presentación del nuevo saber contextualizado a las necesidades del estudiante y su realidad social; organización del trabajo del estudiante; el procesamiento de la información por los estudiantes; la sistematización o construcción del nuevo saber; y la puesta en práctica de lo aprendido. Es el momento donde se evidencia el aprendizaje cognitivo, procedimental y actitudinal que contribuye a fortalecer el proceso de enseñanza del docente y el proceso de aprendizaje del estudiante.

Finalmente, precisa Imbernon Francesc, ${ }^{10}$ respecto a la actividad de salida, que

La salida de la sesión también tiene su importancia. Una buena clase (introducción y desarrollo) puede perderse por una mala salida de ella. Se ha de intentar salir de la sesión con tranquilidad, sin prisas, sin provocar un estrés a todo el grupo cuando corremos en los últimos minutos porque el tiempo se nos ha echado encima y queremos decir muchas cosas.

De igual manera, Eduardo M. Pacheco Peña y Sanyorei Porras Cosme ${ }^{11}$ señalan que la actividad de cierre debe ser entendida como un proceso de mejora continua teniendo en cuenta la evaluación del aprendizaje esperado, las actividades de reforzamiento, y las actividades de extensión. Es el momento de la reflexión de la forma en que aprenden los estudiantes y de cómo enseńa el docente: resumiendo las ideas más importantes, evaluando la forma como aprenden, dando las indicaciones de la siguiente clase, y precisando las tareas, trabajos y evaluaciones del contenido de la asignatura.

Lo indicado líneas anteriores, nos lleva a referirnos a Castillo Arredondo Santiago y Cabrerizo Diago Jesús, ${ }^{12}$ quienes señalan respecto a la evaluación cognitiva que

para evaluar adecuadamente contenidos conceptuales se hace necesario realizar una evaluación inicial que permita al profesor determinar los conocimientos previos y conocer de este modo el nivel de todos y cada uno de los alumnos en cada materia, lo que ha de servir como punto de partida para desarrollar aprendizajes posteriores.

De igual manera, Castillo Arredondo Santiago y Cabrerizo Diago Jesús $(2010)^{13}$ señalan, respecto a la evaluación procedimental, que:

9 Eduardo M. Pacheco Peña y Sanyorei Porras Cosme, op. cit., 81-82.

10 Francesc Imbernon, op. cit., 17.

11 Eduardo M. Pacheco Peńa y Sanyorei Porras Cosme, op. cit., 82.

12 Santiago Castillo Arredondo y Jesús Cabrerizo Diago, Evaluación educativa de aprendizajes y competencias (España: Pearson Educación S. A., 2010), 139.

13 Ibid., 143. 
La evaluación del proceso de aprendizaje de los contenidos procedimentales requiere de la utilización de pruebas o ejercicios que impliquen poner en práctica los procedimientos adquiridos, tanto en situaciones conocidas como en situaciones nuevas de aplicación, lo que supone la realización de una programación previa y adecuada, en la que figuren con claridad los criterios para evaluar contenidos procedimentales y la ponderación que se va a otorgar a los mismos en la evaluación global.

Finalmente, Castillo Arredondo Santiago y Cabrerizo Diago Jesús (2010 ${ }^{14}$ señalan, respecto a la evaluación actitudinal, que:

... las actitudes y los valores pueden aprenderse y por lo tanto deben enseñarse y evaluar su logro. Este proceso de formación actitudinal y de educación en valores se realiza desde la escuela, por medio de procesos conscientes (proyectos de formación), y de otros procesos no conscientes relacionados con las actitudes y los valores latentes en el clima de relaciones interpersonales y con las prácticas educativas que se desarrollan en la escuela.

\section{MATERIALES Y MÉTODOS}

\subsection{MATERIALES}

Al finalizar el ciclo académico 2018-I, entre los meses de marzo a junio de 2018 se procedió a realizar una encuesta a ochenta y siete (87) estudiantes del décimo ciclo, matriculados en la asignatura de Métodos Estadísticos para la Investigación: aula 201 con 23 estudiantes, aula 202 con 35 estudiantes, y aula 302 con 29 estudiantes. Estudiantes que procedieron a evaluar el proceso de enseńanza-aprendizaje en el aula desarrollado por el docente encargado, quienes expresaron, mediante un cuestionario, sus vivencias y experiencias didácticas ocurridas en el aula durante el ciclo académico concluido.

Del total de ochenta y siete (87) estudiantes, se encuestaron a un total de setenta y ocho (78) estudiantes, que representaban el $89,65 \%$ de la población a ser encuestada. Teniendo en cuenta la muestra probabilística, debieron ser encuestados un total de setenta y un (71) estudiantes, pero en realidad se encuestaron a setenta y ocho (78) estudiantes. Esto teniendo en cuenta a E. Luna y A. Torquemada, ${ }^{15}$ quienes, haciendo referencia a McKeachie, precisan que "actualmente, la evaluación de la docencia con base en los cuestionarios de opinión de los estudiantes es reconocida como la estrategia de evaluación con mayor conocimiento conceptual y empírico acumulado en el contexto universitario".

14 Ibid., 146.

15 E. Luna y A. Torquemada, "Los cuestionarios de evaluación de la docencia por los alumnos: balance y perspectivas de su agenda”, Revista Electrónica de Investigación Educativa, Especial (2008): 4, acceso el 21 de junio de 1018, http://redie. uabc.mx/NumEsp1/contenido-lunatorquemada.html 
De tal manera que la técnica empleada fue la encuesta y la fuente documental. El instrumento utilizado fue un cuestionario estructurado con una escala tipo Likert, el mismo que fue elaborado e implementado por el docente responsable de la asignatura a fin de identificar, definir y medir la variable "Proceso de enseñanza-aprendizaje en el aula", teniendo en cuenta las fases de planificación, ejecución y evaluación, las mismas que fueron evaluadas desde la variable "Percepción del estudiante de educación superior", teniendo en cuenta las vivencias y experiencias ocurridas en el ciclo académico y en los tres momentos de las sesiones de clases: actividad de inicio, actividad de proceso y actividad de salida. En ese orden, el cuestionario tuvo veintiún (21) ítems que fueron distribuidos de la siguiente manera:

- Planificación (actividad de inicio)

$$
\begin{aligned}
& 01,02,03,04,05,06 \text { у } 07 \\
& 08,09,10,11,12,13 \text { у } 14 \\
& 15,16,17,18,19,20 \text { у } 21
\end{aligned}
$$

- Desarrollo (actividad de proceso)

- Evaluación (actividad de salida)

\subsection{MÉTODOS}

La investigación fue aplicada teniendo en cuenta que "es aquella que está orientada a resolver objetivamente los problemas de los procesos de producción, distribución, circulación y consumo de bienes y servicios de cualquier actividad humana” ${ }^{16}$ Más aún, cuando la investigación llevada a cabo "se sustenta en la investigación teórica; su finalidad específica es aplicar las teorías existentes a la producción de normas y procedimientos tecnológicos, para controlar situaciones o procesos de la realidad". ${ }^{17}$ En consecuencia, la investigación buscó evaluar la realidad objetiva respecto al proceso de enseñanza- aprendizaje en el aula a fin deconstruir, modificar y actuar sobre dicha realidad que requiere de una adecuación al nuevo escenario social, educativo, científico y tecnológico que atraviesan las universidades del país.

El método empleado fue el hipotético-deductivo en la medida que la investigación realizada "consiste en un procedimiento que parte de algunas aseveraciones en calidad de hipótesis y busca refutar o falsear tales hipótesis, deduciendo de ellas conclusiones generales que deben confrontarse con los hechos antes de constituirse en teorías". ${ }^{18}$ Esto quiere decir, como lo reafirma H. Naupas Paitán y otros, ${ }^{19}$ que "el método hipotético-deductivo consiste en ir de la hipótesis a la deducción para determinar la verdad o falsedad de los hechos, procesos o conocimientos mediante el principio de la falsación".

16 Humberto Naupas Paitán, Elías Mejía Mejía, Eliana Novoa Ramírez y Alberto Villagómez, Metodología de la investigación cuantitativa-cualitativa y redacción de tesis, 4a ed. (Bogotá, Colombia: Ediciones de la U, 2014), 93.

17 Santiago Valderrama Mendoza, Pasos para elaborar proyecto de investigación cientifica cuantitativa, cualitativa y mixta, $5^{\text {a }}$ reimp. (Lima, Perú: Editorial San Marcos E. I. R. L., 2015), 39.

18 César Augusto Bernal Torres, Metodología de la investigación, $4^{\mathrm{a}}$ ed. (Bogotá, Colombia: Pearson Educación de Colombia S. A. S., 2016), 71.

19 Humberto Naupas Paitán et. al., op. cit., 136. 
El enfoque fue cuantitativo en la medida que la investigación se caracterizó “... por utilizar métodos y técnicas cuantitativas y por ende tiene que ver con la medición, el uso de magnitudes, la observación y medición de las unidades de análisis, el muestreo, el tratamiento estadístico". ${ }^{20}$ En ese ordenamiento, se aprecia que la investigación llevada a cabo “... utiliza la recolección de datos y el análisis de los mismos para contestar preguntas de investigación y probar hipótesis formuladas previamente, además confía en la medición de variables e instrumento de investigación, con el uso de la estadística descriptiva e inferencial, en tratamiento estadístico y la prueba de hipótesis; la formulación de hipótesis estadísticas, el diseño formalizado de los tipos de investigación...". ${ }^{21}$

En ese orden de ideas, la investigación tuvo las siguientes características: 1) Según la intervención del investigador fue observacional, ya que no existió intervención del investigador y los datos que se recogieron reflejaron la evolución natural de los hechos, ajenos a la voluntad del investigador. En razón de que “... la observación es la forma más sistematizada y lógica para el registro visual y verificable de lo que se pretende conocer; es decir, es captar de la manera más objetiva posible, lo que ocurre en el mundo real, ya sea para describirlo, analizarlo o explicarlo desde una perspectiva científica”.22 En consecuencia “... el hecho en estudio debe ser considerado como un acontecimiento de la realidad observada, el cual contempla elementos abstractos (teoría) y elementos prácticos (empíricos) para el observador. Por lo tanto, un hecho no es solo un producto de cualquier cosa o situación observada, sino es parte de una realidad y representa un objeto y/o una circunstancia conocida o factible de conocer". ${ }^{23}$

Otra característica fue que 2) Según la planificación de la toma de datos fue prospectiva, ya que los datos necesarios para el estudio fueron recogidos a propósito de la investigación, y 3) Según el número de ocasiones en que se midió la variable de estudio fue transversal, ya que las variables se midieron en una sola ocasión. Finalmente, 4) Según el número de variables de interés, fue analítica, por lo mismo que el análisis estadístico fue bivariado (evaluación de la enseńanza-aprendizaje y percepción del estudiante); donde se plantearon las hipótesis que se pusieron a prueba mediante la asociación, contrastación y explicación.

El nivel de investigación fue explicativo, como lo señalan Roberto Hernández Sampieri, Carlos Fernández Collado y María del Pilar Baptista Lucio ${ }^{24}$ en la medida que "los estudios explicativos van más allá de la descripción de concepto o fenómenos o del establecimiento de

20 Ibid., 97.

21 Ibid.

22 Guillermo Campos y Covarrubias y Nallely Emma Lule Martínez, "La observación, un método para el estudio de la realidad", Xihmai VII, n. ${ }^{\circ} 13$ (enero - junio 2012): 49.

23 Ibid., 50.

24 Roberto Hernández Sampieri, Carlos Fernández Collado y María del Pilar Baptista Lucio, Metodología de la investigación cientifica, $5^{\text {a }}$ ed. (Lima, Perú: McGraw - Hill Educación, 2010), 83-84. 
relaciones entre conceptos; es decir, están dirigidos a responder por las causas de los eventos y fenómenos físicos o sociales. Como su nombre lo indica, su interés se centra en explicar por qué ocurre un fenómeno y en qué condiciones se manifiesta, o por qué se relacionan dos o más variables".

El diseño de investigación fue no experimental y se validó el instrumento en la medida que “... se trata de estudios donde no hacemos variar en forma intencional las variables independientes para ver su efecto sobre otras variables", es decir que "lo que hacemos en la investigación no experimental es observar fenómenos tal como se dan en su contexto natural, para posteriormente analizarlos". ${ }^{25}$ En dicho contexto, "las inferencias sobre las relaciones entre variables se realizan sin intervención o influencia directa, y dichas relaciones se observan tal como se han dado en su contexto natural". ${ }^{26}$ En ese sentido el diseño fue:

$$
\begin{aligned}
& \text { M1: Ox1 Oy1 } \rightarrow \\
& \text { M1 = Estudiantes. } \quad \text { Ox1 = Evaluación del proceso E - A. } \rightarrow \\
& \text { = Índice de C-E. Oy1 = Percepción del estudiante. }
\end{aligned}
$$

Se aplicó el coeficiente Alfa de Cronbach utilizando el Software SPSS 22, a fin de establecer la consistencia interna del instrumento; del cual se aprecia que el resultado fue de un valor de 0,972 , lo que indica que la consistencia interna del instrumento fue alta o excelente. Si se tiene en cuenta la consistencia interna del total de los elementos del instrumento de medición, se aprecia que la consistencia interna del instrumento oscila entre el valor mínimo de 0,970 al valor máximo de 0,972 , lo que indica que la consistencia interna de los ítems del instrumento es alta o excelente.

Respecto a la correlación de las variables en estudio se empleó la prueba de Rho de Spearman, en el cual se aprecia que el valor de las dimensiones de las variables oscila entre 0,775, 0,786 y 0,805 , lo que indica que la correlación entre las dimensiones de la variable evaluación de la enseñanza-aprendizaje en el aula con la percepción del estudiante de educación superior es alta; es decir, la asociación es significativa.

25 Ibid., 149.

26 Ibid., 150. 


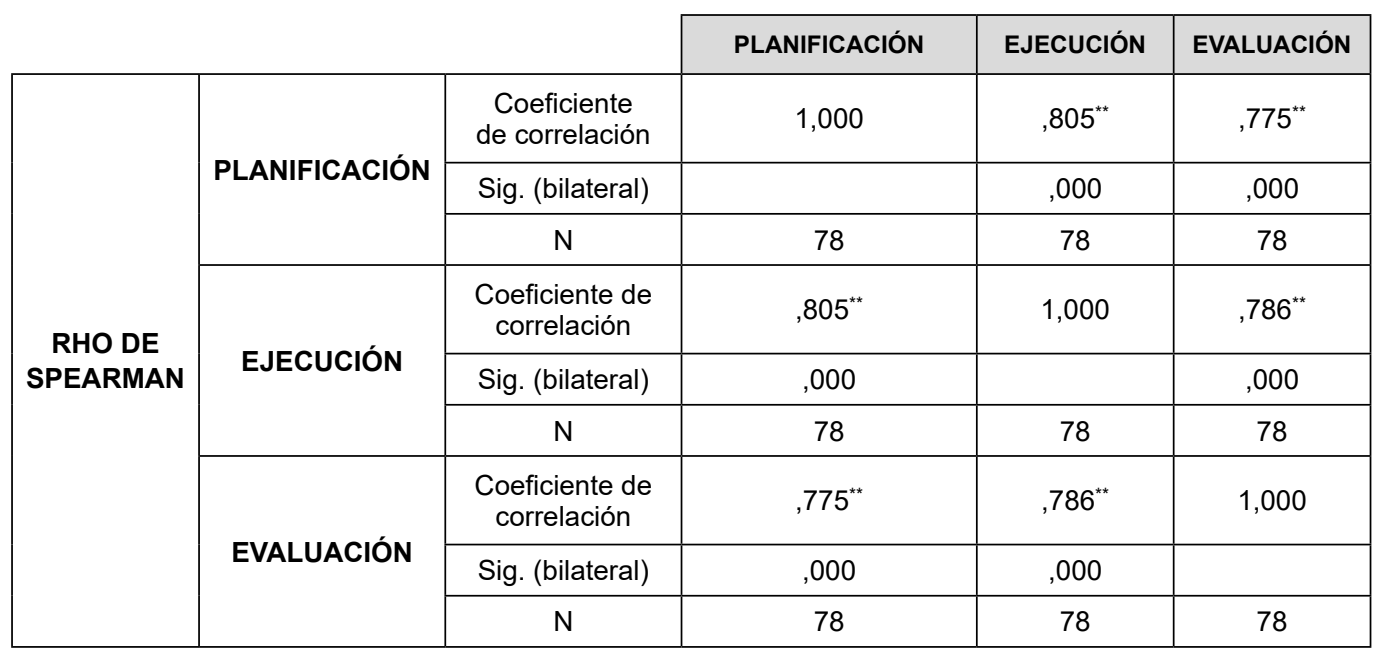

En cuanto a la contrastación de hipótesis, se estimó que la máxima cantidad de error que se estaba dispuesto a aceptar para dar como válida la hipótesis del investigador fue de 0,05 , para lo cual se calculó el p-valor (sig. asintótica) para establecer el límite de error que se está dispuesto a aceptar, y cuyo resultado fue:

a) Respecto a la contrastación de la variable Evaluación de la enseñanza-aprendizaje en el aula con la variable Percepción del estudiante, se aprecia una relación significativa en los veintiún (21) ítems, cuyo valor fue de 0,000 menor al valor de 0,05 que se estaba dispuesto a aceptar.

b) Respecto a la dimensión Planificación de la enseñanza-aprendizaje en el aula se aprecia una relación significativa con la variable Percepción del estudiante de educación superior. En la medida que los siete (07) ítems obtuvieron un valor de 0,000 menor al valor de 0,05 que se estaba dispuesto a aceptar.

\begin{tabular}{|c|c|c|c|c|c|c|c|}
\hline VARIABLE & \multicolumn{7}{|c|}{ EVALUACIÓN ENSEÑANZA-APRENDIZAJE EN EL AULA } \\
\hline DIMENSIÓN & \multicolumn{7}{|c|}{ PLANIFICACIÓN DE LA ENSEÑANZA-APRENDIZAJE } \\
\hline ÍTEMS & P1 & P2 & P3 & P4 & P5 & P6 & P7 \\
\hline & $\begin{array}{l}\text { Asiste } \\
\text { planificadamente } \\
\text { a dictar las clases. }\end{array}$ & \begin{tabular}{|l|} 
Registra \\
asistencia del \\
estudiante \\
en forma \\
planificada.
\end{tabular} & $\begin{array}{l}\text { Explica el } \\
\text { objetivo } \\
\text { planificado } \\
\text { de la clase. }\end{array}$ & \begin{tabular}{|l|} 
Recoge \\
planificadamente \\
saberes previos \\
del estudiante.
\end{tabular} & $\begin{array}{l}\text { Respeta la } \\
\text { planificación } \\
\text { del contenido } \\
\text { silábico. }\end{array}$ & $\begin{array}{l}\text { Desarrolla } \\
\text { el sílabo } \\
\text { siguiendo una } \\
\text { planificación. }\end{array}$ & $\begin{array}{l}\text { Cumple } \\
\text { planificadamente } \\
\text { el horario de } \\
\text { clase. }\end{array}$ \\
\hline Chi-cuadrado & $161,897^{a}$ & $128,051^{a}$ & $140,333^{b}$ & $50,103^{a}$ & $123,128^{a}$ & $183,538^{b}$ & $229,692^{b}$ \\
\hline gl & 3 & 3 & 4 & 3 & 3 & 4 & 4 \\
\hline Sig. asintótica & 000 & 000 &, 000 &, 000 &, 000 &, 000 &, 000 \\
\hline
\end{tabular}


c) Respecto a la dimensión Ejecución de la enseñanza-aprendizaje en el aula se aprecia una relación significativa con la variable Percepción del estudiante de educación superior. En la medida que los siete (07) ítems obtuvieron un valor de 0.000 menor al valor de 0,05 que se estaba dispuesto a aceptar.

\begin{tabular}{|c|c|c|c|c|c|c|c|}
\hline VARIABLE & \multicolumn{7}{|c|}{ EVALUACIÓN ENSEÑANZA-APRENDIZAJE EN EL AULA } \\
\hline DIMENSIÓN & \multicolumn{7}{|c|}{ EJECUCIÓN DE LA ENSEÑANZA-APRENDIZAJE } \\
\hline ÍTEMS & P8 & P9 & P10 & P11 & P12 & P13 & P14 \\
\hline & $\begin{array}{l}\text { Desarrolla el } \\
\text { contenido de } \\
\text { la asignatura } \\
\text { con fluidez. }\end{array}$ & $\begin{array}{l}\text { Desarrolla los } \\
\text { conocimientos de } \\
\text { manera sencilla y } \\
\text { clara. }\end{array}$ & $\begin{array}{l}\text { Desarrolla los } \\
\text { conceptos } \\
\text { con ejemplos } \\
\text { prácticos. }\end{array}$ & $\begin{array}{l}\text { Desarrolla la } \\
\text { motivación } \\
\text { en forma } \\
\text { permanente. }\end{array}$ & $\begin{array}{l}\text { Desarrolla } \\
\text { la utilidad e } \\
\text { importancia } \\
\text { del contenido. }\end{array}$ & $\begin{array}{l}\text { Desarrolla la } \\
\text { comunicación } \\
\text { en un clima de } \\
\text { confianza. }\end{array}$ & $\begin{array}{l}\text { Desarrolla y } \\
\text { absuelve la } \\
\text { pregunta del } \\
\text { estudiante. }\end{array}$ \\
\hline Chi-cuadrado & $149,179^{b}$ & $148,795^{\mathrm{b}}$ & $108,026^{b}$ & $130,846^{b}$ & $89,077^{a}$ & $125,974^{\mathrm{b}}$ & $117,897^{b}$ \\
\hline gl & 4 & 4 & 4 & 4 & 3 & 4 & 4 \\
\hline Sig. asintótica &, 000 &, 000 &, 000 &, 000 & ,000 &, 000 &, 000 \\
\hline
\end{tabular}

d) Respecto a la dimensión Evaluación de la enseñanza-aprendizaje en el aula se aprecia una relación significativa con la Percepción del estudiante de educación superior. En la medida que los siete (07) ítems obtuvieron un valor de 0,000 menor al valor de 0,05 que se estaba dispuesto a aceptar.

\begin{tabular}{|c|c|c|c|c|c|c|c|}
\hline VARIABLE & \multicolumn{7}{|c|}{ EVALUACIÓN ENSEÑANZA-APRENDIZAJE EN EL AULA } \\
\hline DIMENSIÓN & \multicolumn{7}{|c|}{ EVALUACIÓN DE LA ENSEÑANZA-APRENDIZAJE } \\
\hline ÍTEMS & P15 & P16 & P17 & P18 & P19 & P20 & P21 \\
\hline & $\begin{array}{l}\text { El material } \\
\text { entregado es } \\
\text { útil para la } \\
\text { evaluación. }\end{array}$ & $\begin{array}{l}\text { La evaluación } \\
\text { se ajusta al } \\
\text { contenido del } \\
\text { sílabo }\end{array}$ & $\begin{array}{l}\text { Evaluación } \\
\text { promueve } \\
\text { aprendizaje } \\
\text { teórico- } \\
\text { cognitivo. }\end{array}$ & $\begin{array}{l}\text { Evaluación } \\
\text { promueve } \\
\text { aprendizaje } \\
\text { práctico- } \\
\text { procedimental. }\end{array}$ & $\begin{array}{l}\text { Evaluación } \\
\text { refuerza } \\
\text { aprendizaje } \\
\text { en la } \\
\text { investigación }\end{array}$ & $\begin{array}{l}\text { Entrega la } \\
\text { evaluación } \\
\text { oportunamente. }\end{array}$ & $\begin{array}{l}\text { Evaluación } \\
\text { fortalece } \\
\text { comprensión } \\
\text { de los temas } \\
\text { tratados. }\end{array}$ \\
\hline Chi-cuadrado & $110,513^{a}$ & $133,179^{a}$ & $158,282^{b}$ & $159,949^{b}$ & $106,923^{a}$ & $75,000^{c}$ & $75,000^{c}$ \\
\hline gl & 3 & 3 & 4 & 4 & 3 & 2 & 2 \\
\hline Sig. asintótica & ,000 & 000 &, 000 &, 000 &, 000 & 000 & .000 \\
\hline
\end{tabular}

Se confirmó que el valor calculado del KMO del análisis factorial confirmatorio fue igual 0,870, lo que indica que la adecuación del muestreo alcanzó un nivel aceptable y por lo tanto el modelo factorial empleado es efectivo. ${ }^{27}$ Del mismo modo, se confirmó mediante la prueba de esfericidad de Bartlett $^{28}$ que por ser la significancia igual a 0,00 inferior al valor de 0,05,

27 Instituto Nacional de Estadística e Informática, Guía para la aplicación del análisis multivariado a las encuestas de hogares (Lima, Perú: Dirección Técnica de Demografía e Indicadores Sociales, 2002), 26.

28 Ibid. 
se rechaza la hipótesis nula $\left(\mathrm{H}_{0}\right)$ y se acepta la hipótesis alterna $\left(\mathrm{H}_{1}\right)$. En consecuencia, se concluye que es poco probable que la matriz de correlación sea una matriz de identidad; por lo tanto, el coeficiente de correlación es estadísticamente significativo.

Se elaboró la matriz de comunalidad, donde se evidencia que todos los parámetros presentan valores mayores a 0,4 y se concluye que todos los parámetros son importantes para la explicación del problema. El valor máximo fue de 0,921, lo que significa que el $92 \%$ de la variabilidad es explicada por los tres factores; mientras que el valor mínimo fue de 0,578 , lo que significa que el $58 \%$ de la variabilidad es explicada por los tres factores.

Se confirmó en la matriz de la varianza total explicada ${ }^{29}$ del análisis factorial confirmatorio, en la cual se aprecia que los tres primeros factores son los únicos que cumplen con las especificaciones, y explican el 79,77 \% del comportamiento de la varianza total explicada. Por tanto, el 20,23\% del comportamiento de la varianza total es explicada por otros factores.

De igual manera se elaboró la matriz de componentes rotados en la medida que "es una herramienta importante para la interpretación de los factores. Así, se giran en el origen los ejes de referencia de los factores hasta alcanzar una determinada posición" . ${ }^{30}$ Teniendo en cuenta los resultados de la matriz de componentes rotados, se muestra la disposición de las variables en los factores según el grado de importancia: Factor $01=0,793+0,792+0,773+0,753+$ $0,714+0,680+0,657+0,636+0,566 ;$ Factor $02=0,843+0,840+0,827+0,807+0,796$ $+0,791+0,749+0,563$; y Factor $03=0,846+0,783+0,779+0,691$.

Esto nos lleva a explicar la evaluación de la enseñanza-aprendizaje en el aula y la percepción del estudiante de educación superior, de la siguiente manera:

Factor 01 = Está representado por la ejecución de la enseñanza-aprendizaje en el aula que está referida a la comunicación en un clima de confianza; el desarrollo del sílabo siguiendo una planificación ;el desarrollo de los conceptos con ejemplos prácticos; el desarrollo del contenido con fluidez; el desarrollo de los conocimientos de manera sencilla y clara; el cumplimiento planificado del horario de clases; el recojo de los saberes previos del estudiante; el desarrollo de la motivación en forma permanente; y el desarrollo de la utilidad e importancia del contenido; que orienta las actividades de proceso en el aula. El mismo que explica el 30,00 $\%$ de la percepción del estudiante de educación superior, que tiene sobre las actividades de proceso que se desarrolla en el aula.

29 J. Mejía Trejo, Las ciencias de la administración y el análisis multivariante. Proyectos de investigación, análisis y discusión de resultados (México: Universidad de Guadalajara, 2017): 21.

30 Ibid., 24. 
Factor 02 = Está representado por la evaluación de la enseńanza-aprendizaje en el aula que está referido a que la evaluación refuerza el aprendizaje en la investigación; el material entregado es útil para la evaluación; la entrega oportuna de la evaluación; la evaluación se ajusta al contenido del sílabo; la evaluación fortalece la comprensión de los temas tratados; la evaluación promueve el aprendizaje teórico-cognitivo; la evaluación promueve el aprendizaje práctico-procedimental; y el docente desarrolla y absuelve la pregunta del estudiante; que orienta las actividades de salida en el aula. El mismo que explica el 29,15\% de la percepción del estudiante de educación superior que tiene sobre las actividades de salida que se desarrolla en el aula.

Factor 03 = Dicho factor está representado por la planificación de la enseñanza-aprendizaje en el aula que está referido a la asistencia planificada en el dictado de clases; el registro planificado de la asistencia del estudiante; el respeto planificado del contenido silábico; y la explicación del objetivo de la sesión de clase; que orienta las actividades de inicio en el aula. El mismo que explica el 20,62 \% de la percepción del estudiante de educación superior que tiene sobre las actividades de inicio que se desarrollan en el aula.

\section{RESULTADOS}

Respecto a la variable evaluación de la enseñanza-aprendizaje en el aula, el $74 \%$ de estudiantes opinaban que era muy eficiente; el $19 \%$ que era eficiente; el $3 \%$ que era regular; el $2 \%$ que era deficiente; y el $2 \%$ que era muy deficiente.

\section{GRÁFICO 1: EVALUACIÓN ENSEÑANZA-APRENDIZAJE EN EL AULA}

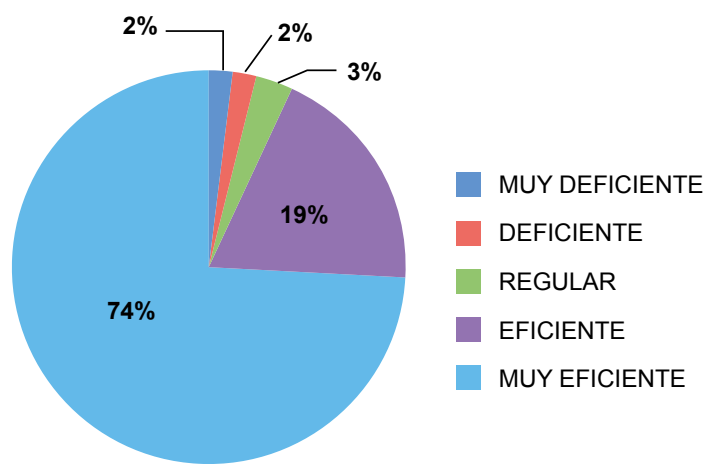

Respecto a la dimensión planificación de la enseńanza-aprendizaje en el aula, el $77 \%$ de estudiantes opinaban que era muy eficiente; el $16 \%$ que era eficiente; el $3 \%$ que era regular; el $2 \%$ que era muy deficiente; y el $2 \%$ que era deficiente. 


\section{GRÁFICO 2: DIMENSIÓN PLANIFICACIÓN}

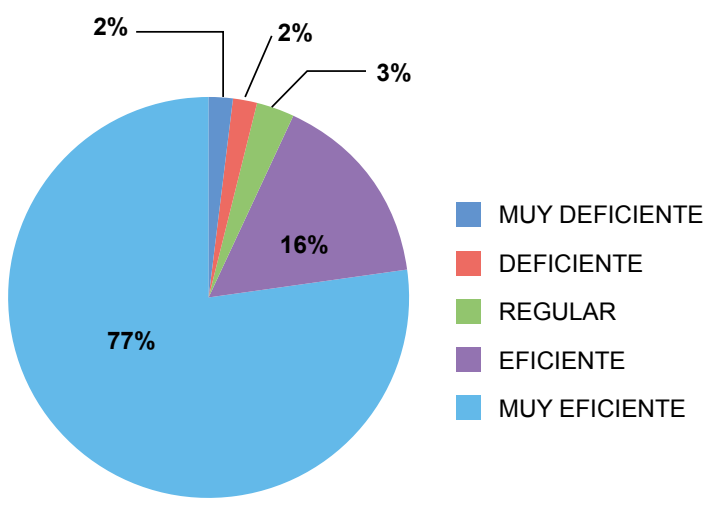

Respecto a la dimensión desarrollo de la enseñanza-aprendizaje en el aula, el 69 \% de estudiantes opinaban que era muy eficiente; el $23 \%$ que era eficiente; el $4 \%$ que era regular; el $2 \%$ que era deficiente; y el $2 \%$ que era muy deficiente.

GRÁFICO 3: DIMENSIÓN DESARROLLO

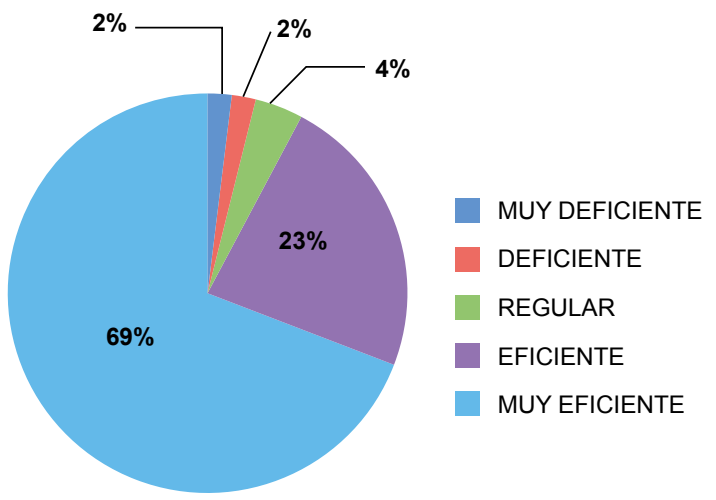

Respecto a la dimensión evaluación de la enseñanza-aprendizaje en el aula, el 77 \% opinaba que era muy eficiente; el $18 \%$ que era eficiente; el $3 \%$ que era regular; el $1 \%$ que era muy deficiente; y el $1 \%$ que era deficiente. 
GRÁFICO 4: DIMENSIÓN EVALUACIÓN

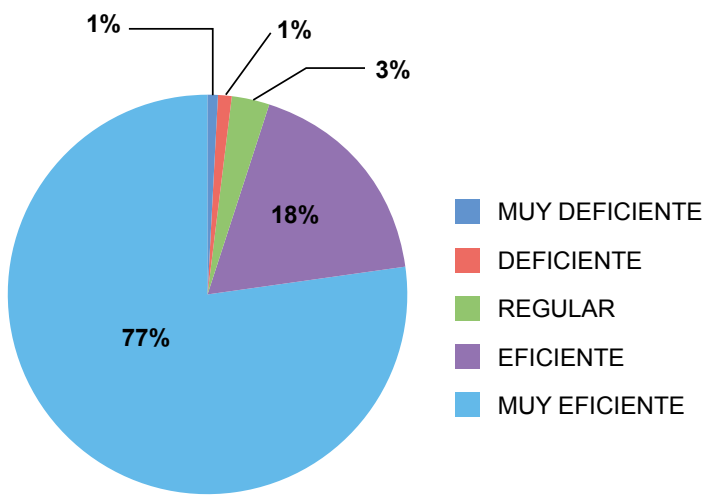

\section{CONCLUSIONES}

Se evidencia que la variable evaluación de la enseñanza-aprendizaje en el aula es explicada significativamente en un 79,77\% por la variable percepción del estudiante de educación superior.

Se evidencia que la dimensión planificación de la enseñanza-aprendizaje en el aula es explicada significativamente en un 20,62 \% por la variable percepción del estudiante de educación superior.

Se evidencia que la dimensión ejecución de la enseñanza-aprendizaje en el aula es explicada significativamente en un 30,00 \% por la variable percepción del estudiante de educación superior.

Se evidencia que la dimensión evaluación de la enseñanza-aprendizaje en el aula es explicada significativamente en un $29,15 \%$ por la variable percepción del estudiante de educación superior.

\section{RECOMENDACIONES}

La evaluación de la enseñanza-aprendizaje en el aula debe ser entendida por el docente como un proceso formativo que busca mejorar la calidad del servicio educativo que se brinda en el aula; que contribuye a superar las debilidades y corregir los errores que descubren los estudiantes en los docentes para convertirlos en fortalezas y oportunidades; con el propósito de mejorar la calidad de enseñar a aprender al estudiante por parte del docente y fortalecer la tarea del estudiante de aprender a aprender en educación superior. 
La planificación de la enseñanza-aprendizaje en el aula debe ser entendida como la organización planificada de las sesiones de clases en el aula que integra la actividad de inicio; la misma que debe ser entendida como un proceso de mejora continua en la organización planificada de las sesiones de clases; que tenga en cuenta la problematización planeada desde la realidad del estudiante; la motivación inicial que capte la atención inicial del estudiante; la revisión de los saberes previos del estudiante; el conflicto cognitivo que genere un debate entre los estudiantes; y el propósito de la sesión que implica dar a conocer los aprendizajes que se espera que logren, el tipo de actividad que van a realizar y cómo serán evaluados.

La ejecución de la enseñanza-aprendizaje en el aula debe ser entendida como la presentación del nuevo saber contextualizado a las necesidades del estudiante y su realidad social que integra la actividad de proceso; la misma que debe ser entendida como un proceso de mejora continua de la contextualización del nuevo saber a las necesidades del estudiante y realidad social; organización del trabajo del estudiante; el procesamiento de la información; la sistematización o construcción del nuevo saber; y la puesta en práctica de lo aprendido. Es el momento donde se evidencia el aprendizaje cognitivo, procedimental y actitudinal que contribuye a fortalecer el proceso de enseńanza del docente y el proceso de aprendizaje del estudiante.

La evaluación de la enseñanza-aprendizaje en el aula debe ser entendida como la evaluación del aprendizaje esperado, las actividades de reforzamiento y las actividades de extensión que integra la actividad de cierre; la misma que debe ser entendida como el momento de la reflexión de la forma en que aprenden los estudiantes y de cómo enseña el docente; resumiendo las ideas más importantes; evaluando la forma como aprenden; señalando las actividades de extensión; precisando la tarea, trabajo y evaluación del contenido de la sesión.

\section{REFERENCIAS}

- Bernal Torres, César Augusto. Metodología de la investigación. 4a ed. Bogotá, Colombia: Pearson Educación de Colombia S. A. S., 2016.

- Campos y Covarrubias, Guillermo; y Nallely Emma Lule Martínez. "La observación, un método para el estudio de la realidad”. Xihmai VII, n. 13 (enero - junio 2012): 45-60.

- Castillo Arredondo, Santiago y Jesús Cabrerizo Diago. Evaluación educativa de aprendizajes y competencias. España: Pearson Educación S. A., 2010.

- Hernández Sampieri, Roberto; Carlos Fernández Collado y María del Pilar Baptista Lucio. Metodología de la investigación cientifica. $5^{\text {a }}$ ed. Lima, Perú: McGraw - Hill Educación, 2010 . 
- Imbernon, Francesc. Mejorar la enseñanza y el aprendizaje en la Universidad. $1^{1}$ ed. Barcelona, Espańa: Ediciones Octaedro, 2009.

- Instituto Nacional de Estadística e Informática. Guía para la aplicación del análisis multivariado a las encuestas de hogares. Lima, Perú: Dirección Técnica de Demografía e Indicadores Sociales, 2002.

- Luna, E.; y A. Torquemada. "Los cuestionarios de evaluación de la docencia por los alumnos: balance y perspectivas de su agenda". Revista Electrónica de Investigación Educativa, Especial (2008). Acceso el 21 de junio de 1018. http://redie.uabc.mx/NumEsp1/contenido-lunatorquemada.html

- Mejía Mejía, Elías. Metodología de la investigación cientifica. $1^{a}$ ed. Lima: Universidad Nacional Mayor de San Marcos, Facultad de Educación, Unidad de Postgrado, 2005.

- Mejía Trejo, J. Las ciencias de la administración y el análisis multivariante. Proyectos de investigación, análisis y discusión de resultados. México: Universidad de Guadalajara, 2017.

- Naupas Paitán, Humberto; Elías Mejía Mejía, Eliana Novoa Ramírez y Alberto Villagómez. Metodología de la investigación cuantitativa-cualitativa y redacción de tesis. $4^{\mathrm{a}}$ ed. Bogotá, Colombia: Ediciones de la U, 2014.

- Pacheco Peña, Eduardo M.; y Sanyorei Porras Cosme. "Los momentos de la sesión a través de las rutas de aprendizaje. Propuesta de trabajo para los alumnos de las carreras de Educación Secundaria de la UNDAC”. Horizonte de la Ciencia 4, n. ${ }^{\circ} 7$ (diciembre 2014).https://doi.org/10.26490/uncp.horizonteciencia.2014.7.113

- Solar R., María Inés; y Claudio Díaz L. "Los procesos de enseñanza-aprendizaje en el aula universitaria: una mirada desde las creencias de académicos de trabajo social y periodismo". Estudios Pedagógicos XXXV, n. ${ }^{\circ} 1$ (2009): 181-197.

https://doi.org/10.4067/S0718-07052009000100011

- Soubal Caballero, Santos. "La gestión del aprendizaje. Algunas preguntas y respuestas sobre en relación con el desarrollo del pensamiento en los estudiantes". Polis 7, n. 21 (2008): 311-337. https://doi.org/10.4067/S0718-65682008000200015(2008)

- Valderrama Mendoza, Santiago. Pasos para elaborar proyecto de investigación cientifica cuantitativa, cualitativa y mixta. 5a reimp. Lima, Perú: Editorial San Marcos E. I. R. L., 2015.

RECIBIDO: $10 / 07 / 2018$

APROBADO: $15 / 09 / 2018$ 


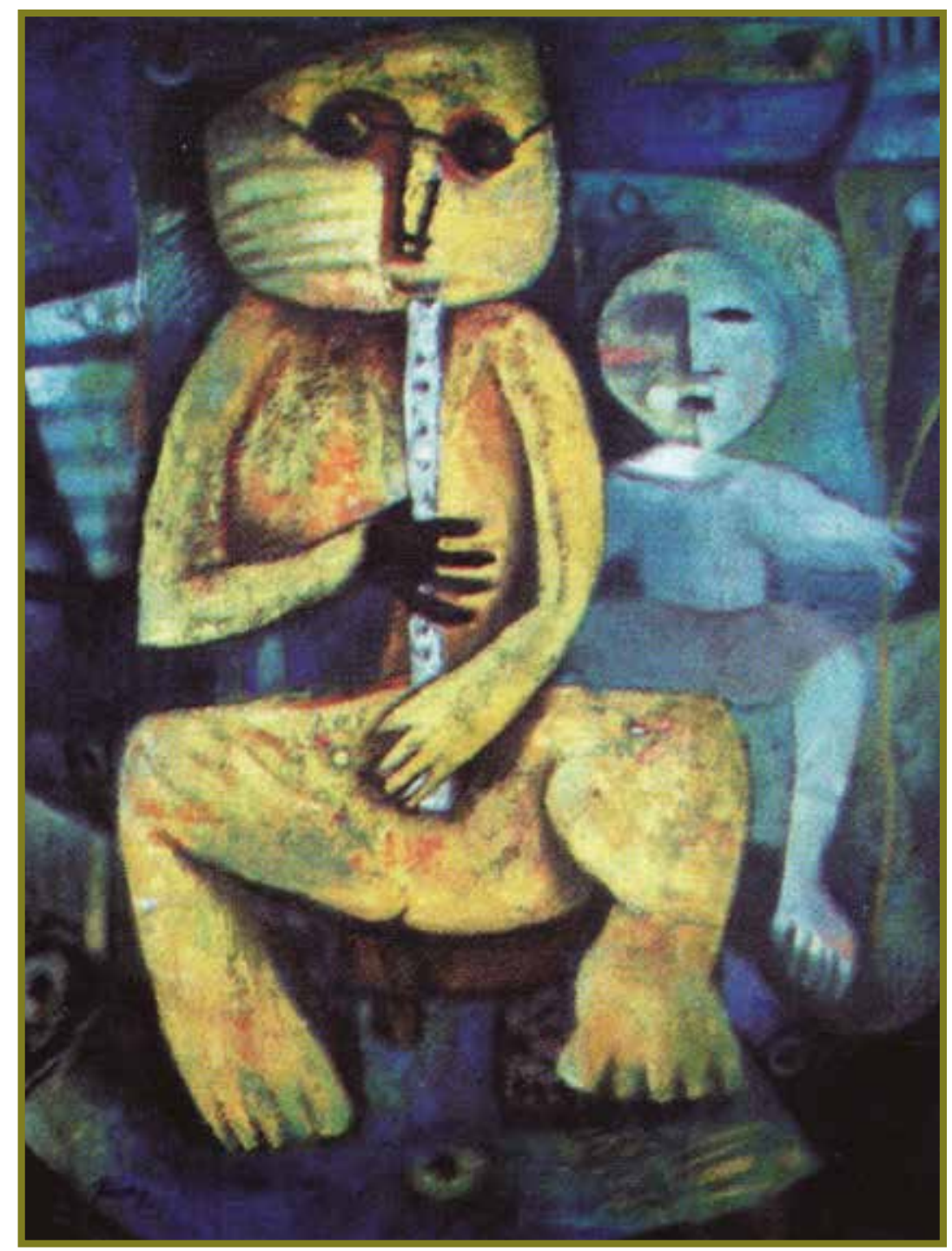

Sonidos de Esperanza. Pintura. Juan Carlos Ñañake. 